\title{
Progress in the Integrated Simulation of Thermal-Hydraulic Operation of the ITER Magnet System
}

\author{
M. Bagnasco, D. Bessette, L. Bottura, C. Marinucci, and C. Rosso
}

\begin{abstract}
A new integrated computer code is being developed for the simulations of the overall behavior of the ITER magnet cryo-system. The existing THEA, FLOWER and POWER codes, assembled as modules of a computational environment (SuperMagnet) have been upgraded to perform global simulations of the cooling circuit for the ITER magnet system.

The thermal coupling resulting from the generic geometric configurations has been implemented to realize quasi-three-dimensional simulations of the winding pack. In this paper we present details on the model.
\end{abstract}

Index Terms-Heat transfer, superconducting magnets, thermal-hydraulics.

\section{INTRODUCTION}

$\mathbf{I}$ N RECENT years the increasing importance gained by the superconducting magnet system in the frame of the ITER project, brought to the development of a large set of computational tools [1]-[6] with different degrees of complexity and various targets, to assist and verify the design of superconducting magnets as well to perform the analysis of experimental data during the preparatory $R \& D$ phase. The added value of the most advanced codes is their capability to simulate simultaneously different aspects of the operation of a superconducting coil but the cost to achieve it is typically an increased complexity in the problem definition [6] or the necessity to adapt very different codes to run within a standard software manager [7].

Starting from the existing tools, the SuperMagnet suite [8] has been created by CryoSoft with the intent to bring together the computer codes already developed in house [3]-[5] each simulating a part of the global system, the added value being the dedicated software environment in which every code can

Manuscript received October 20, 2009. First published April 08, 2010; current version published May 28, 2010. This work was supported by the European Community under the Contract of Association between EURATOM/Swiss, was carried out within the framework of the European Fusion Development Agreement. The views and opinions expressed herein do not necessarily reflect those of the European Commission.

M. Bagnasco and C. Marinucci are with EPFL-CRPP Fusion Technology, 5232 Villigen PSI, Switzerland (e-mail: Maurizio.Bagnasco@psi.ch).

D. Bessette is with ITER Organisation, 13108 St. Paul Les Durance, France (e-mail: Denis.Bessette@iter.org).

L. Bottura is with CERN, AT-MSC, 1211 Geneva, Switzerland (e-mail: Luca. Bottura@cern.ch).

C. Rosso is with CryoSoft, France.

Color versions of one or more of the figures in this paper are available online at http://ieeexplore.ieee.org.

Digital Object Identifier 10.1109/TASC.2010.2043836 transfer and receive data from/to the processes run in parallel by the software manager itself.

The main components of the SuperMagnet are the following:

- THEA (Thermal-Hydraulic Electric Analysis): a multi-physics tool for the analysis of superconducting cables which combines the modeling of heat conduction in solids (strands, structural components), heat transfer in the coolant by means of one-dimensional (1D) Euler-like conservation equation, and the electric behavior of the conductor accounting for resistive and/or inductive current distribution;

- FLOWER: a tool for the simulation of standard components of cryogenic circuits (heat exchangers, pumps, turbines, etc.) combined in a hydraulic network representative of the proximity cryogenics of a magnet;

- POWER: a tool for the simulation of electrical networks, to which the superconducting component is connected and of which it is part.

The above codes exist as stand-alone applications, and have been modified to integrate them into the SuperMagnet suite. In addition, a new tool has been prototyped, HEATER, to account for the heat conduction in winding packs and structures, which could not be else directly included in the above mentioned codes, as described later. The SuperMagnet external environment provides the glue among these tools, launching the requested codes (or multiple instances of the same code with different input data), managing the coupling between different codes, providing the correct data exchange between every running process and performing the interpolation whenever needed to match requested and available data. The main working concept of the top level manager is shown schematically in Fig. 1.

\section{NEW HEATER MODULE}

\section{A. Model Description}

In order to extend the 1D modeling capabilities of the different codes, a new module (HEATER) has been conceived. Its main goal is to simulate the actual heat transfer paths especially in case of pancake or layer wound conductors as well as heat loads coming from support structures and/or external sources (e.g. nuclear heating). The heat conduction equation

$$
\rho c_{p}(T) \frac{\partial T(x, y, z)}{\partial t}-\nabla \cdot(k(T) \nabla T(x, y, z))=Q(x, y, z)
$$

where $T$ is the temperature, $k$ is the material thermal conductivity and $Q$ the external heat source, is solved on the selected 


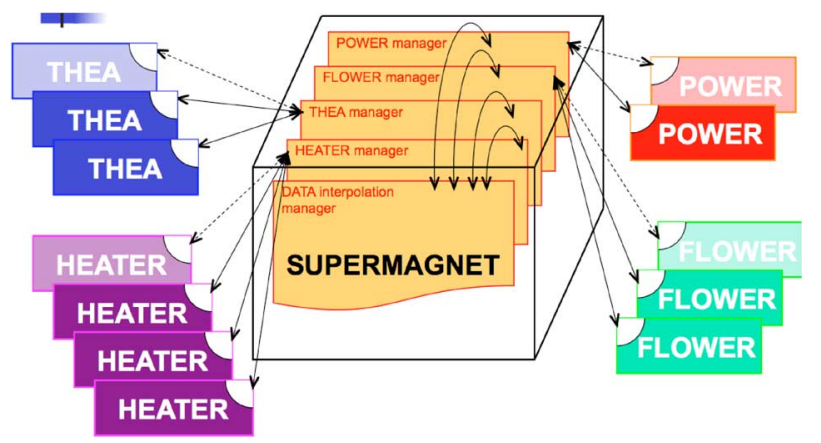

Fig. 1. Conceptual scheme of the SuperMagnet software environment. The single processes dialogate with the corresponding manager inside SuperMagnet and the internal data interpolation manager collects and dispatches the requested data from/to all the application managers.

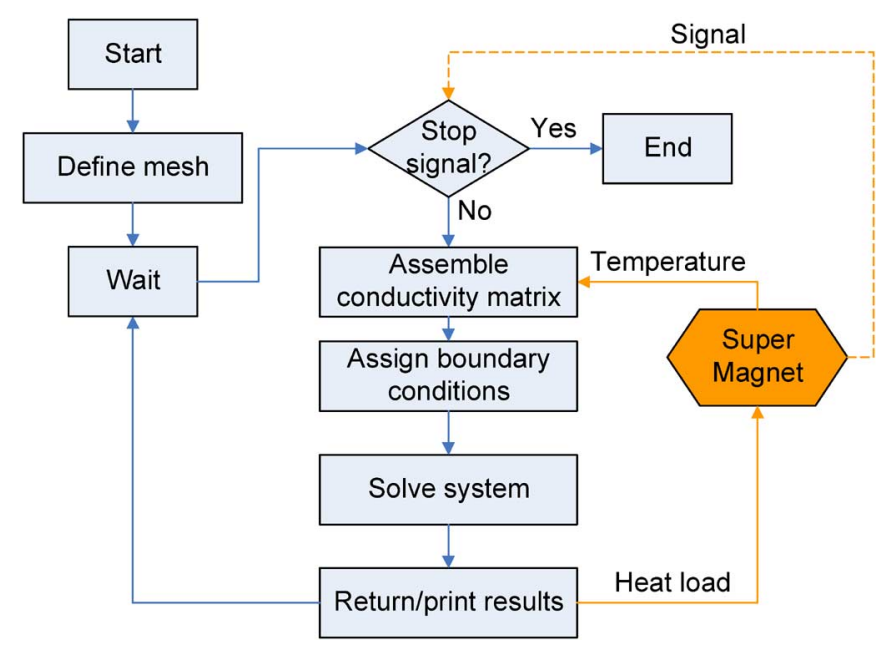

Fig. 2. HEATER flowchart.

domain. The conceptual flowchart of the HEATER module is sketched in Fig. 2. The discretization is performed with user defined Finite Elements (FE) meshes consisting of one-, two- or three-dimensional elements provided to the code in a dedicated section of the input file with the node coordinates and the element boundaries and properties (e.g. material).

The conduction matrix is built considering the material properties provided in the mesh definition according to the example (in 1D) shown in Fig. 3. Starting from node "A", the 1D elements simulate the chain of thermal resistances of the conductor, the jacket and the insulation, respectively. Since the temperatures at which the properties are computed are expected to vary during the simulation, the conduction matrix is recalculated at each computation step.

Concerning the boundary conditions, temperature and/or heat loads can be imposed on user defined sets of nodes and/or elements like lines, surfaces or volumes to model the coupling with warmer structures or perform stand-alone HEATER runs e.g. assuming a given temperature profile along the conductor axes.

\section{B. Coupling With Other Processes}

Inside the HEATER module, the coupling with other external processes like THEA or FLOWER is performed by defining a

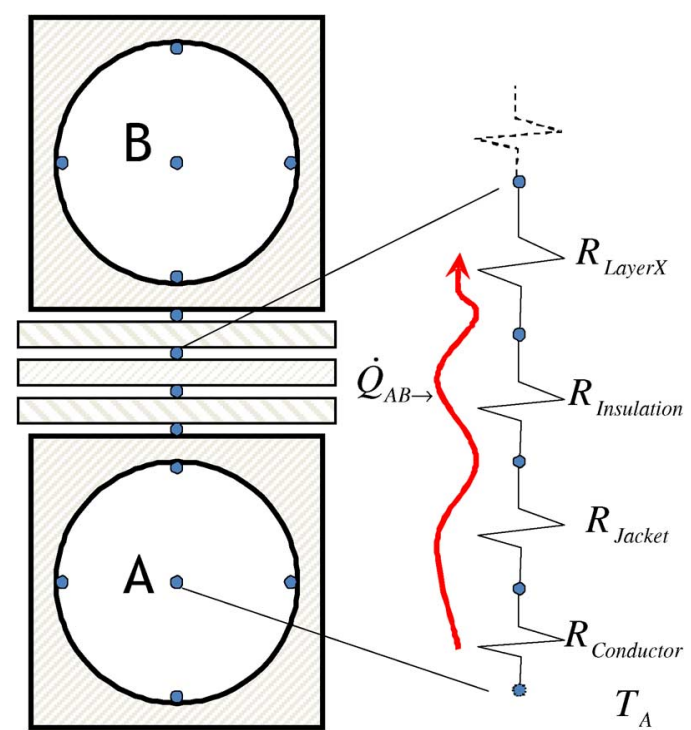

Fig. 3. Generation of the thermal resistance $(R)$ chain from the axis location "A" to the axis location "B". Only half of the chain $\left(R_{\text {He-Jacket }}, R_{\text {Jacket }}, R_{\text {Insulation }}, \ldots\right)$ is sketched from the description of the conductor cross section on the left hand side. "Layer-X" may represent inter-turn insulation, inter-pancake insulation as well as any other material on the modeled heat transfer direction.

specific set of nodes and/or element as "external". This means that boundary conditions for the heat conduction equation will be provided by SuperMagnet after inquiring the corresponding process defined as linked to that set in the SuperMagnet input file.

As an example, in the HEATER mesh represented in Fig. 4(a) the "external" type nodes are highlighted as well as the "external" lines and are retained in Fig. 4(b). The nodes can be in principle linked (see Fig. 4(b)) not only to single locations in a separate THEA object but also to FLOWER volumes. The "external" line type can be on the other hand arbitrarily linked to any THEA (solid or coolant) or FLOWER object.

\section{An Example}

To show an example of application of SuperMagnet with the new HEATER module an ITER CS-like coil with three pancakes and five turns per pancake (total length $\sim 46 \mathrm{~m}$ per pancake) is modeled with a circle in square stainless steel jacket with an inner diameter of $38 \mathrm{~mm}$, an outer dimension of $42 \mathrm{~mm}$ and an arbitrary insulation layer of $0.2 \mathrm{~mm}$ thickness. The cross section of the coil, as modeled with the FE mesh, is shown in Fig. 5 and the same structure is repeated 12 times to represent the triple pancake. Since the pancakes are hydraulically independent, the nodes in the center of each conductor are linked to form three lines, which are eventually linked to the coolant flowing in three separate THEA runs (one per pancake). The THEA simulations are then linked to each other through the HEATER jacket and insulation elements.

It is assumed that a current of $45 \mathrm{kA}$ flows in the coil in a constant background magnetic field of $13 \mathrm{~T}$. At the simulation start a heat pulse of $10 \mathrm{~ms}$ is given at $x=21 \mathrm{~m}$ in the central pancake so that a quench is initiated. The current is then ramped down after $50 \mathrm{~ms}$ with a time constant of $2 \mathrm{~s}$ and the quench evolution 

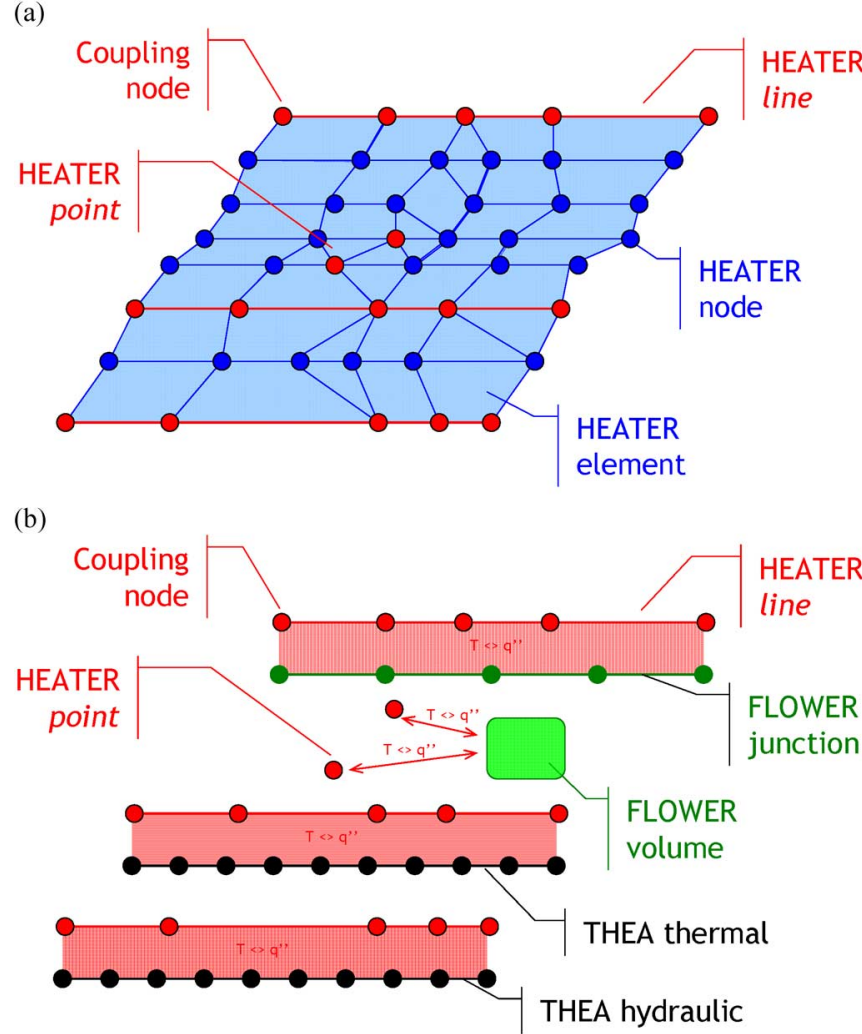

Fig. 4. (a) Definition of HEATER components (node, element) and coupling possibilities (point, line). (b) On the same mesh as above, examples of coupling with THEA and FLOWER components. See main text for details.

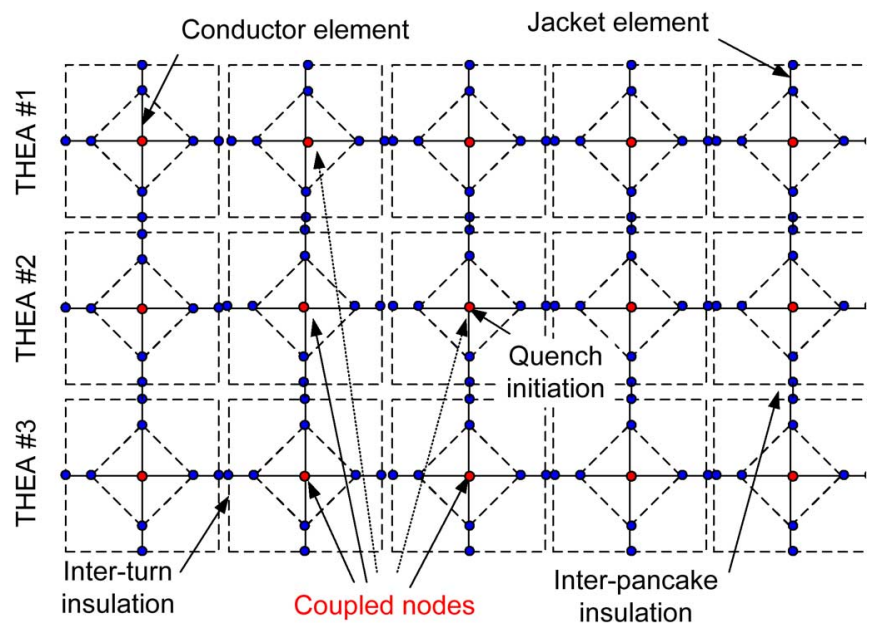

Fig. 5. Cross section of the CS-like triple pancake at $\mathrm{x}=21 \mathrm{~m}$. The solid lines represent the elements between two nodes. The dashed lines are only a guide to the eye. The highlighted nodes at the center of each conductor are linked to separate THEA simulations. The position of the quench initiation is also shown.

is observed. Please note that due to the different winding direction of the pancakes, the temperature is not expected to rise at the same axial location in all the pancakes.

The results plotted in Fig. 6 show that after $20 \mathrm{~s}$ the heat conduction through jacket and insulation is such that its effects are to be seen not only in the quenched pancake temperature profile but also in the neighboring ones.
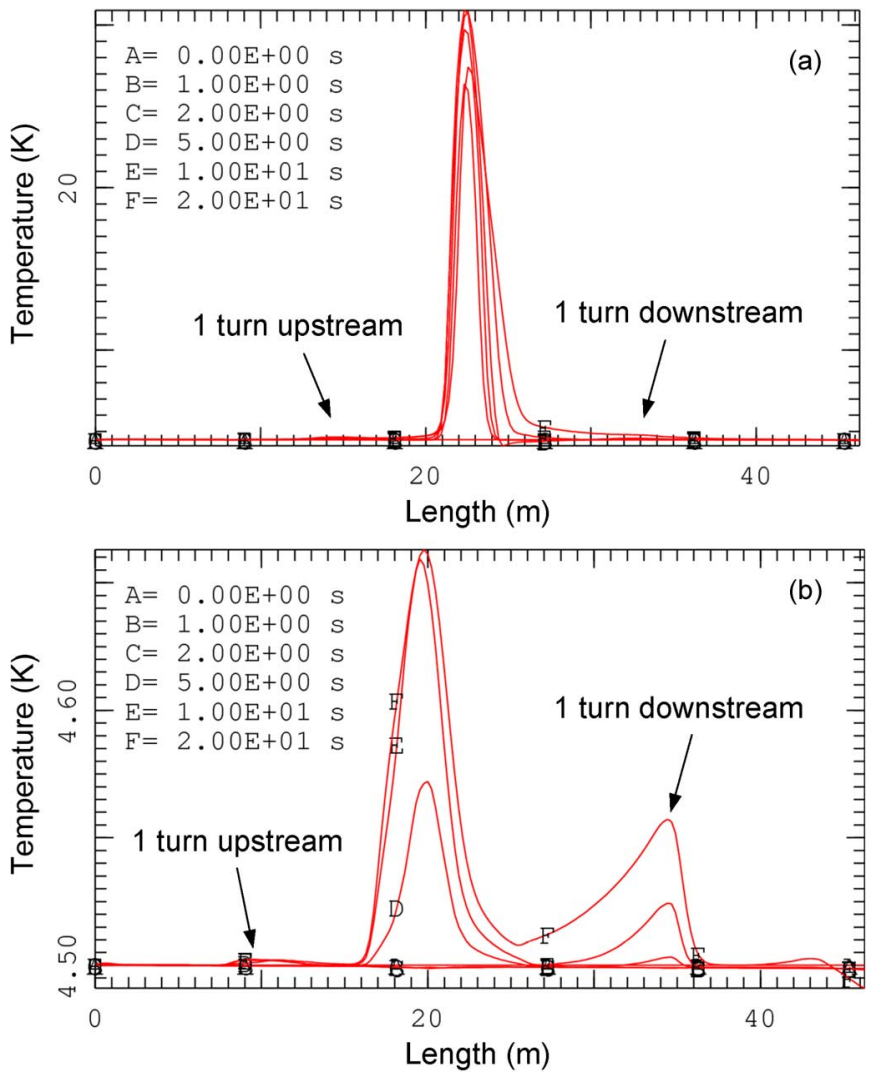

Fig. 6. Temperature axial profiles along the pancake (a) \#2 and (b) \#1. Evidences of heat conduction through the insulation after $20 \mathrm{~s}$ simulation can be seen in all pancakes at both positions one turn upstream and downstream.

\section{Performance ScAling}

The target of the SuperMagnet suite is the simulation of a real ITER magnet system, which includes up to hundreds of conductors. The data transfers that have to be managed clearly increase with the number of processes needed and therefore a study was performed to assess the limits of the SuperMagnetoperating system interaction. The test was run on an Intel Xeon E5450 equipped with $2 \times 3.0 \mathrm{GHz}$ Quad Core, i.e. 8 cores total) and $2 \mathrm{~GB}$ RAM per core (=16 GB total). The operating system was Scientific Linux.

A simple circuit is modeled by FLOWER consisting of two volumes connected by an increasing number of conductors modeled with THEA. We have monitored the total CPU time required to complete the run, as a function of the number of THEA processes (the computational time of FLOWER is negligible with respect to THEA). From the trend shown in Fig. 7 it is clear how the performance takes advantage of the intrinsic parallel architecture of SuperMagnet (every code is launched every time as a single, independent process) as long as the number of processes is lower than the number of cores ( 8 in this case). Above this limit, after a reproducible bump around 16 THEA processes (twice the number of cores), probably due to lags in the shared processes among the cores, the performance scales quite linearly and reaches an upper hardware dependent limit where the available memory does not allow anymore addressing properly all the required data. 


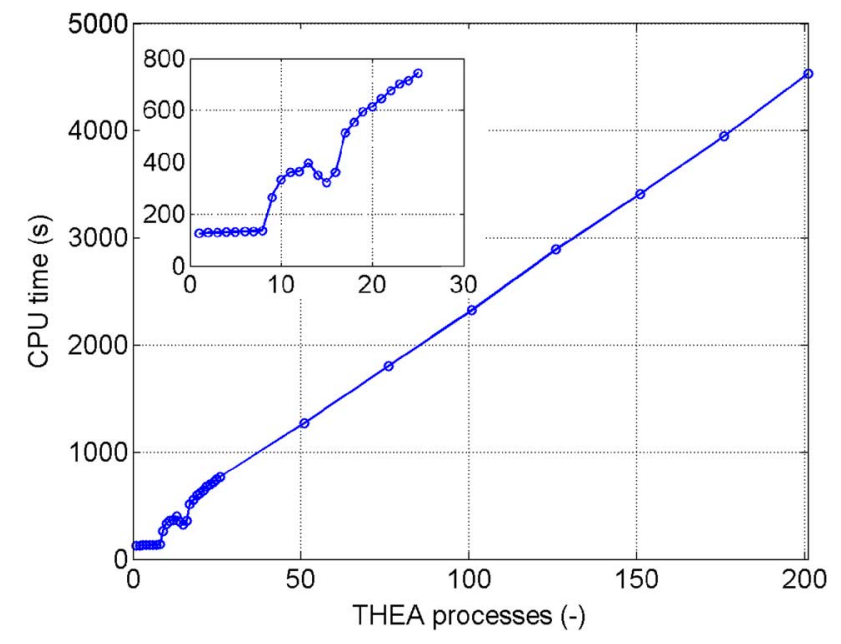

Fig. 7 Execution time as a function of the number of THEA processes and zoom on the range from 1 to 25 .

It must be however stressed that an arbitrary number of conductors can be grouped in single FLOWER or THEA runs and therefore the limiting figure above is relevant only in view of the easy scalability of the code, which allows to create very large runs merging, as an example, separated smaller scale simulations into more general simulations.

\section{CONClusion}

A new module to account for multidimensional heat transfer has been developed and coupled to the already existing modules for the SuperMagnet computer code suite. The capability to realize complex thermal-hydraulical and electrical simulations not only qualifies the SuperMagnet suite for the verification of the ITER magnet system design, but the ability to create arbitrary meshes to evaluate heat transfer between different objects also sports its application with different magnet types like, e.g., adiabatic magnets, bath cooled magnets and accelerator magnets.

\section{ACKNOWLEDGMENT}

The authors thank the technical support of Paul Scherrer Institut (PSI).

\section{REFERENCES}

[1] L. Bottura, "A numerical model for the simulation of quench in the ITER magnets," J. Comp. Phys., vol. 125, pp. 26-41, 1996.

[2] R. Zanino, S. De Palo, and L. Bottura, "A two-fluid code for the thermohydraulic transient analysis of CICC superconducting magnets," $J$. Fus. Energy, vol. 14, no. 1, pp. 25-40, 1995.

[3] L. Bottura and C. Rosso, "Flower, a model for the analysis of hydraulic networks and processes," Cryogenics, vol. 43, no. 3-5, pp. 215-224, 2003.

[4] POWER, Electric Network Simulation of Magnetic Systems ver. 2.1, August 2009

[5] L. Bottura, C. Rosso, and M. Breschi, "A general model for thermal, hydraulic, and electric analysis of superconducting cables," Cryogenics, vol. 40, no. 8-10, pp. 617-626, 2000.

[6] D. Bessette, N. Shatil, and E. Zapretilina, "Simulations of the ITER toroidal field coil operation with the VINCENTA code," IEEE Trans. Appl Supercond, vol. 16, pp. 795-798, 2006.

[7] L. Savoldi Richard, F. Casella, B. Fiori, and R. Zanino, "Development of the cryogenic circuit conductor and coil (4C) code for thermalhydraulic modeling of ITER superconducting coils," in Proceedings of the 22nd International Cryogenic Conference (ICEC22), 2009, pp. 619-624.

[8] SuperMagnet, Multitasking Code Manager ver. 2.0, September 2009. 